\title{
GENERALIZED HYPERANALYTIC FUNCTION THEORY
}

\author{
BY ROBERT P. GILBERT ${ }^{1}$ AND GERALD N. HILE
}

Communicated by M. H. Protter, April 3, 1972

Lipman Bers [1] and Ilya Vekua [2] extended the concept of an analytic function by considering the weak solutions of elliptic systems of two equations, with two unknowns, and two independent variables. The solutions they investigated have come to be known as generalized (or pseudo) analytic functions. Subsequently Avron Douglis [3] introduced a class of functions which satisfy (classically) the principal part of an elliptic system of $2 r$ equations, with $2 r$ unknowns, and two independent variables. These functions are known as hyperanalytic functions. The present paper extends the class of functions studied by Douglis in much the same way as Bers and Vekua have extended the analytic functions. Hence, we refer to our class of functions as generalized hyperanalytic functions.

We shall be concerned with elliptic systems in two variables of the form

$$
\begin{gathered}
u_{0, x}-v_{0, y}+p_{0,0} u_{0}+q_{0,0} v_{0}=0, \\
u_{0, y}+v_{0, x}+r_{0,0} u_{0}+s_{0,0} v_{0}=0, \\
u_{k, x}-v_{k, y}+a u_{k-1, x}+b u_{k-1, y}+\sum_{l=0}^{k}\left(p_{k l} u_{l}+q_{k l} v_{l}\right)=0, \\
u_{k, y}+v_{k, x}+a v_{k-1, x}+b v_{k-1, y}+\sum_{l=0}^{k}\left(r_{k l} u_{l}+s_{k l} v_{l}\right)=0, \\
k=1, \ldots, r-1 .
\end{gathered}
$$

Our work uses the hypercomplex function theory developed by A. Douglis [3] for the special case of (1) where only the principal part appears.

Douglis introduced the commutative algebra over the reals generated by the two elements $i$ and $e$, subject to the multiplication rules

$$
i^{2}=-1, \quad i e=e i, \quad e^{r}=0 .
$$

A hypercomplex number in this algebra has the form

AMS 1969 subject classifications. Primary 3081, 3544, 3520.

Key words and phrases. Generalized analytic mappings, pseudo-analytic function of Bers, Vekua, etc., elliptic systems (linear), generalized solutions.

${ }^{1}$ Research sponsored by the Air Force Office of Scientific Research, Air Force Systems Command, USAF, under AFOSR Grant Nos. 12067-67 and 71-2205A. The United States Government is authorized to reproduce and distribute reprints for Government purposes notwithstanding any copyright notation hereon. 


$$
c=\sum_{k=0}^{r-1} c_{k} e^{k}
$$

where each $c_{k}$ is an ordinary complex number. A hypercomplex function $w$ of two variables is written as

$$
w(x, y)=\sum_{k=0}^{r-1} w_{k}(x, y) e^{k}
$$

where each $w_{k}$ is complex-valued. By use of the operator

$$
D=D_{x}+i D_{y}+e a D_{x}+e b D_{y},
$$

and the identifications $w_{k}=u_{k}+i v_{k}$, the principal part of system (1) can be written as

$$
D w=0 .
$$

Douglis has shown that solutions of (2) retain many properties of the analytic functions in complex variable theory.

We extend Douglis' theory to more general systems. With proper definitions of the complex functions $A_{k l}$ and $B_{k l}$, (1) can be written as the hypercomplex equation

$$
D w+\sum_{k=0}^{r-1} e^{k} \sum_{l=0}^{k}\left(A_{k l} w_{l}+B_{k l} \bar{w}_{l}\right)=0 .
$$

Here derivatives are in the Sobolev sense. We also treat a more restrictive special case of (3):

$$
D w+A w+B \bar{w}=0,
$$

where in this formula $A$ and $B$ are hypercomplex functions. When $r=1$, equation (4) reduces to the complex equation for which Bers and Vekua have developed an extensive theory.

Introducing some variations on the techniques of Vekua, we have been able to prove the following theorems. (Detailed proofs are to be given elsewhere.)

THEOREM I. Let $a$ and $b$, along with their first partial derivatives, be bounded and Hölder-continuous in the whole plane $E$, and also lie in $L_{p}(E)$ for some $p, 1 \leqq p<2$. Let each $A_{k l}, B_{x l}$ lie in $L_{p, 2}(E)$ for some $p>2$. If $w$ satisfies (3) in E, then

(i) either the zeros of $w$ are isolated, or $w \equiv 0$.

(ii) If $w$ is continuous and bounded in the whole plane, then

$$
w(z)=c \exp \{\omega(z)\},
$$

where $c$ is a hypercomplex constant, and $\omega$ is a hypercomplex valued func- 
tion, Hölder-continuous in $E$, which is $O\left(|z|^{(2-p) / p}\right)$ at infinity. The exponential function in this formula generalizes the exponential function in complex variable theory.

(iii) (Liouville's Theorem.) If $w$ is continuous and bounded in the whole plane, and vanishes at a point $z_{0}$, then $w \equiv 0$.

(iv) (Generating pairs.) Continuous and bounded solutions $w$ of (3), for fixed $A_{k l}, B_{k l}$, have the representation

$$
w(z)=c_{0} \varphi_{0}(z)+c_{1} \varphi_{1}(z),
$$

where $c_{0}$ and $c_{1}$ are hypercomplex constants, and $\varphi_{0}$ and $\varphi_{1}$, which depend on the $A_{k l}, B_{k l}$, are also bounded and continuous solutions.

THEOREM II. Let $\mathbf{5}$ be a bounded domain whose boundary $\Gamma$ consists of a finite number of simple, piecewise smooth, Jordan curves. Let $a, b, A$ and $B$ have the same properties as in Theorem I. Suppose $w$ satisfies (4) in $\mathbf{G}$ and is continuous in $\mathbb{E}$. Then

(i) $w$ has a "Cauchy integral" representation in terms of its boundary values and the boundary values of two "fundamental kernels" $\Omega_{1}$ and $\Omega_{2}$, namely

$$
w(z)=\frac{1}{2 \pi i} \int_{\Gamma} \Omega_{1}(z, \zeta) w(\zeta) d t(\zeta)-\Omega_{2}(z, \zeta) \overline{w(\zeta)} \overline{d t(\zeta)},
$$

for $z \in \mathfrak{G}$, where $t(\zeta)$ is a so-called "generating solution" of (2). The fundamental kernels depend only on $A$ and $B$ (and $a$ and $b$ ) and the domain (5.

(ii) $w$ may also be expressed by an integral formula involving the boundary values of the fundamental kernels and boundary values of a hyperanalytic function $\Phi$ (satisfying (2)) associated with w, namely

$$
w(z)=\frac{1}{2 \pi i} \int_{\Gamma} \Omega_{1}(z, \zeta) \Phi(\zeta) d t(\zeta)-\Omega_{2}(z, \zeta) \overline{\Phi(\zeta)} \overline{d t(\zeta)} .
$$

(iii) An alternate representation for $w$ in terms of the hyperanalytic function $\Phi$ is given by

$$
\begin{aligned}
w(z) & =\mathscr{K}(\Phi ; \mathfrak{5}) \\
& \equiv \Phi(z)+\int_{\mathfrak{G}} \int_{1} \Gamma_{1}\left(z, \zeta ;(\mathfrak{5}) \Phi(\zeta) d \xi d \eta+\int_{\mathfrak{F}} \int_{2} \Gamma_{2}(z, \zeta ; \mathfrak{( 5 )} \overline{\Phi(\zeta)} d \xi d \eta,\right.
\end{aligned}
$$

where

$$
\begin{aligned}
& \Gamma_{1}\left(z, \zeta ;(5) \equiv \frac{1}{2 \pi i} \frac{t_{x}}{i+e b} D_{\zeta} \Omega_{1}(z, \zeta),\right. \\
& \Gamma_{2}\left(z, \zeta ;(5) \equiv \frac{1}{2 \pi i} \frac{\bar{t}_{x}}{i-e \bar{b}} D_{\bar{\zeta}} \Omega_{2}(z, \zeta) .\right.
\end{aligned}
$$


As a concluding remark, we mention that with these representations we can construct a complete (in the uniform norm) family of solutions to (4) bounded and continuous in the closure of $(5$. This procedure uses a correspondence given by Douglis, between solutions of (2) and analytic functions.

\section{REFERENCES}

1. L. Bers, Theory of pseudo-analytic functions, Lecture Notes, Institute for Mathematics and Mechanics, New York University, New York, 1953 (mimeographed). MR 15, 211.

2. I. N. Vekua, Generalized analytic functions, Fizmatgiz, Moscow, 1959; English transl., Pergamon Press, London; Addison-Wesley, Reading, Mass., 1962. MR 21 \# 7288; MR 27 \# 321 .

3. A. Douglis, A function-theoretic approach to elliptic systems of equations in two variables, Comm. Pure Appl. Math. 6 (1953), 259-289. MR 16, 257.

Department of Mathematics, Indiana University, Bloomington, Indiana 47401

Current address (G. N. Hile): Department of Mathematics, University of California, Berkeley, California 94720 